\title{
TAFRO Syndrome and Elusive Diagnosis of Idiopathic Multicentric Castleman Disease Treated with Empiric Anti- Interleukin-6 Therapy
}

\author{
Corinne Williams $^{a} \quad$ Alexis Phillips ${ }^{b} \quad$ Vikram Aggarwal $^{c}$ \\ Liron Barnea Slonim $^{d}$ David C. Fajgenbaum ${ }^{e, f}$ Reem Karmalia, $g$ \\ aRobert H. Lurie Comprehensive Cancer Center, Northwestern University, Chicago, IL, \\ USA; bUniversity of Pennsylvania, Philadelphia, PA, USA; 'Division of Nephrology and \\ Hypertension, Northwestern University Feinberg School of Medicine, Chicago, IL, USA; \\ ${ }^{\mathrm{d} D i v i s i o n}$ of Pathology, Northwestern University, Chicago, IL, USA; ${ }^{\mathrm{e}}$ Translational Medicine \& \\ Human Genetics, University of Pennsylvania, Philadelphia, PA, USA; fOrphan Disease Center,

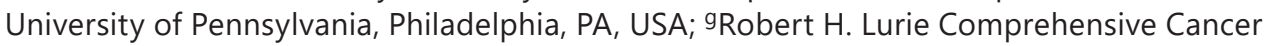 \\ Center, Chicago, IL, USA
}

\section{Keywords}

Idiopathic multicentric Castleman disease $\cdot$ TAFRO $\cdot$ Interleukin- $6 \cdot$ Siltuximab - Treatment

\begin{abstract}
TAFRO syndrome is defined by the presence of thrombocytopenia $(T)$, anasarca $(A)$, fever $(F)$, reticulin fibrosis/renal dysfunction $(R)$, and organomegaly $(O)$ and can be seen with idiopathic multicentric Castleman disease (iMCD) or as an isolated process without iMCD. Although the diagnosis of $\mathrm{M} M C D$ in patients with TAFRO can be challenging to make, iMCD should remain high on the differential diagnosis. Similar to iMCD, the pathophysiology of TAFRO is not well understood but is thought to be related to hypercytokinemia, with interleukin (IL)- 6 playing a pivotal role. Anti-IL- 6 monoclonal antibody therapy is an effective treatment modality for iMCD, but to date, there is no clear guidance on treatment of TAFRO in the absence of definitive diagnosis of $\mathrm{i} M C D$, leading to suboptimal management and high morbidity. We report a case of TAFRO syndrome and demonstrate benefit with the empiric use of anti-IL- 6 antibody therapy in the context of delayed diagnosis of iMCD.
\end{abstract}




\section{Introduction}

Castleman disease (CD) is a heterogeneous group of lymphoproliferative disorders that demonstrate systemic inflammation and share common histopathological features ranging from hyaline vascular to plasmacytic. CD can present as solitary lymph node (LN) enlargement (unicentric CD) or widespread lymphadenopathy (LAD) (multicentric CD; MCD) with or without the presence of HHV-8 infection [1]. HIV- and HHV-8-negative MCD, also known as idiopathic MCD (iMCD), is a rare complex disease with an incidence of $\sim 1,500$ cases/year in the USA [2]. iMCD is characterized by pro-inflammatory hypercytokinemia, resulting in systemic inflammatory symptoms, diffuse LAD, and even multi-organ failure [3].

iMCD is subdivided into patients with TAFRO syndrome (iMCD-TAFRO), that is patients who demonstrate thrombocytopenia (T), anasarca (A), fever (F), reticulin fibrosis/renal dysfunction (R), and organomegaly (O); and patients who do not meet TAFRO criteria (iMCD not otherwise specified, iMCD-NOS). iMCD-NOS patients typically experience thrombocytosis, hypergammaglobulinemia, and a less severe disease course than iMCD-TAFRO [4]. Regardless of subtype, the pathophysiology of iMCD is not well understood but is thought to be related to hypercytokinemia, with interleukin-6 (IL-6) playing a pivotal role in a portion of patients $[2,5]$. While TAFRO syndrome can occur in patients without an established diagnosis of iMCD, iMCD should remain highest on a clinician's differential in all patients newly presenting with TAFRO syndrome. Other diseases reported to demonstrate this syndrome include Sjogren's, lupus, lymphoma, leukemia, and acute viral infections [1]. We present a case of TAFRO with an elusive diagnosis of IMCD and contend that empiric use of anti-IL-6 therapy in such cases may improve patient outcomes.

\section{Case Description}

A 34-year-old woman presented with fevers, thrombocytopenia, acute kidney injury, hypoalbuminemia, and shock of unknown etiology requiring intubation and continuous venovenous hemofiltration. CT imaging showed hepatosplenomegaly with mild generalized LAD. She underwent bone marrow biopsy that showed focal areas of increased fibrosis with increased megakaryocytes and normal morphology. Left axillary core LN biopsy demonstrated polytypic plasmacytosis, but other histopathologic features of iMCD were not conclusively present. Infectious workup including HHV-8 and HIV was negative. She received highdose steroids and IVIG with improvement in renal function and stabilization of blood counts. The patient was discharged on high-dose steroids but subsequently re-admitted with a urinary tract infection and was found to have recurrent thrombocytopenia and worsening renal function. Again, the patient was in a critical condition, this time with fevers and severe anasarca. A renal biopsy demonstrated acute tubular necrosis with vacuolar degeneration and tubulointerstitial disease. Notably, IL-6, sIL-2R, and vascular endothelial growth factor were elevated with normal immunoglobulin, triglyceride, and fibrinogen levels (Table 1). Due to significant thrombocytopenia refractory to transfusion, an excisional LN biopsy could not be safely obtained. Given that other potential underlying autoimmune, neoplastic, and infectious conditions were excluded, she was empirically treated for iMCD-TAFRO in the setting of clinical signs and symptoms characteristic of this process (LAD, AKI, anemia, hypoalbuminemia, elevated IL- 6 and ESR/CRP levels, and normal immunoglobulin levels). High-dose steroids and siltuximab $11 \mathrm{mg} / \mathrm{kg}$ q3 weeks were started with normalization of renal function, CBC, and all other laboratory parameters over several months (shown in Fig. 1A). Serial imaging obtained showed persistent cervical LAD. One year after starting anti-IL-6 therapy, CT scans demonstrated enlarging cervical LNs. Excisional LN biopsy at this time confirmed

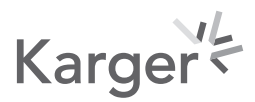




\section{Case Reports in Oncology}

Table 1. Diagnostics and laboratory values for our patient on presentation

\begin{tabular}{l|l}
\hline Case Rep Oncol 2021;14:1359-1365 \\
\hline DOI: 10.1159/000518079 & $\begin{array}{l}\text { @ 2021 The Author(s). Published by S. Karger AG, Basel } \\
\text { www.karger.com/cro }\end{array}$ \\
\hline
\end{tabular}

Williams et al.: Empiric Anti-IL-6 Therapy for TAFRO without iMCD

\begin{tabular}{|c|c|}
\hline Diagnostics & Value [range] \\
\hline $\begin{array}{l}\text { Fever, hepatosplenomegaly, generalized } \\
\text { LAD, anasarca in imaging }\end{array}$ & Yes \\
\hline WBC & 41.4 K/UL [3.5-10.5] \\
\hline Hemoglobin & $5.5 \mathrm{~g} / \mathrm{dL}[11.6-15.4]$ \\
\hline Platelets & 18 K/UL [140-390] \\
\hline Viral studies (HIV, HHV-8, hepatitis C) & Not detected \\
\hline Fibrinogen (max) & 999 mg/dL [200-393] \\
\hline Ferritin (max) & 694 ng/mL [11-307] \\
\hline Triglycerides & $219 \mathrm{mg} / \mathrm{dL}[<100]$ \\
\hline IL-6 & $52 \mathrm{pg} / \mathrm{mL}[<5]$ \\
\hline Soluble IL-2 receptor & $2,898 \mathrm{pg} / \mathrm{mL}$ \\
\hline VEGF & $190 \mathrm{pg} / \mathrm{mL}[<1,033]$ \\
\hline BM biopsy & $\begin{array}{l}\text { + Fibrosis and increased } \\
\text { megakaryocytes }\end{array}$ \\
\hline UPEP & No bands \\
\hline SPEP/IF & No bands \\
\hline Free light chain ratio & $1.97[0.26-1.65]$ \\
\hline Uric acid & Not available \\
\hline QI & $\begin{array}{l}\text { IgG } 839[700-1,600] \\
\text { IgA } 120[70-400] \\
\text { IgM } 41[40-230]\end{array}$ \\
\hline LDH & 397 Unit/L [81-234] \\
\hline Sedimentation rate (max) & $125 \mathrm{~mm} / \mathrm{h}[4-25]$ \\
\hline Albumin & $2.2 \mathrm{~g} / \mathrm{dL}[3.4-5]$ \\
\hline Alkaline phosphatase & 142 Unit/L [46-116] \\
\hline Creatinine (max) & $6.21 \mathrm{mg} / \mathrm{dL}[0.5-1.17]$ \\
\hline
\end{tabular}

IL, interleukin; LAD, lymphadenopathy; BM, bone marrow; VEGF, vascular endothelial growth factor.

CD with hyaline vascular histopathological features (shown in Fig. 1B-F). She has since remained on siltuximab for an additional year without clinical symptoms or laboratory abnormalities.

\section{Discussion/Conclusion}

TAFRO is an inflammatory syndrome identified by the presence of thrombocytopenia, anasarca, fever, reticulin fibrosis, and organomegaly. The syndrome was originally described in 2010 when 3 patients presented with the aforementioned symptom constellation and LAD with CD-like histology [6]. Numerous patients with TAFRO syndrome and CD-like histologies were since identified and treated successfully with steroids and anti-IL-6 antibodies, a standard iMCD treatment regimen. In light of this, many researchers began to consider TAFRO syndrome as a distinct subtype of iMCD, and as such, iMCD was divided into 2 categories: iMCD with TAFRO (iMCD-TAFRO) and iMCD without TAFRO (iMCD-NOS). 
iMCD-TAFRO is distinct from iMCD-NOS in clinical presentation and clinicopathologic features. Characteristic histology of iMCD-TAFRO includes atrophic germinal centers with expansion of the interfollicular zone, highly dense endothelial venules, and few mature plasma cells, whereas iMCD-NOS typically shows plasma cell histology [7]. iMCD without TAFRO typically presents with thrombocytosis, hypergammaglobulinemia, and less severe anasarca. Conversely, iMCD-TAFRO presents with leukocytosis, thrombocytopenia, severe anasarca, elevated alkaline phosphatase, and normal immunoglobulin levels [7]. Patients with iMCDTAFRO are known to have a more aggressive clinical course with worse outcomes than iMCDNOS; some patients develop life-threatening cytokine storm with organ failure and death [2-4].

The current diagnostic criteria for iMCD-TAFRO require LN histology consistent with CD, along with meeting 3 of 5 TAFRO symptoms [8]. As iMCD-TAFRO portends a more aggressive clinical course, prompt diagnosis and treatment are important in minimizing patient mortality. However, the necessity of LN histology confirming CD features in cases with suspicion for iMCD-TAFRO may create barriers to swift management. In patients with TAFRO in the absence of definitive iMCD diagnosis, Masaki's diagnostic criteria should be considered, termed TAFRO syndrome without proven iMCD (TAFRO-w/op-iMCD) [9]. A diagnosis of TAFRO-w/op-iMCD requires 3 major and 2 minor criteria, similar to diagnostic criteria for iMCD-TAFRO, but does not require LN biopsy [9]. In a retrospective analysis of patients with iMCD-TAFRO and TAFRO-w/op-iMCD, the clinical manifestations, laboratory findings, and survival rate were similar, proposing the 2 groups could be considered a single clinical entity, although further research that confirms likeness in underlying biology is needed to validate this proposition [9].

While pathophysiology of both iMCD-TAFRO and iMCD-NOS is not well understood, both are syndromes associated with hypercytokinemia [10]. Excessive activation of inflammatory pathways involving immune cells and the overproduction of pro-inflammatory cytokines such as IL-6 along with vascular endothelial growth factor lead to histopathologic changes in LNs and the systemic symptoms observed in iMCD-TAFRO and iMCD-NOS [11]. Siltuximab, a chimeric immunoglobulin G1k monoclonal antibody that blocks IL-6, is currently approved for the treatment of iMCD [12].

Using our case, we would argue that patients with TAFRO who do not meet criteria for iMCD or who cannot be tested safely for iMCD should still be treated empirically for iMCDTAFRO, barring other causes. While there is currently no standard treatment for TAFRO syndrome in the absence of confirmed iMCD, we propose hypercytokinemia as a driver of clinical manifestations of the syndrome (shown in Fig. 1G) as with iMCD-TAFRO. Accordingly, rational therapeutic options would include corticosteroids, immunosuppressive therapy, rituximab, and/or anti-IL-6 therapies like siltuximab based on their use in iMCD-TAFRO [4].

Fig. 1. a Trends in laboratory parameters in our patient from initial presentation to initiation of anti-IL-6 antibody therapy. b-f LN biopsy in our patient demonstrated pathologic features characteristic of hyaline vascular type CD including $\mathbf{b}$ Regressed germinal center which contains predominantly follicular dendritic cells with relatively few lymphocytes, and the surrounding mantle zone forms concentric rings lined up along FDC processes, imparting an "onion skin" pattern typical of hyaline vascular type CD; c Increased vascularity and relatively preserved architecture with open sinuses, although distinct follicles are not readily identified on H\&E; d Markedly increased plasma cells, located in the interfollicular area as highlighted by CD138; e, f Polytypic plasma cells highlighted by polytypic kappa and lambda in situ hybridization probes. g Proposed mechanisms leading to the signs and symptoms of TAFRO: activation of macrophages, B cells and T cells drive the production of cytokines including IL-6, angiogenic growth factor VEGF, and antibodies, resulting in thrombocytopenia, anasarca, fever, renal dysfunction, fibrosis of the marrow, and organomegaly. Differences in cytokine/chemokine profiles drive variability in clinical symptoms and presentation. CD, Castleman disease; IL, interleukin; LN, lymph node; VEGF, vascular endothelial growth factor. 


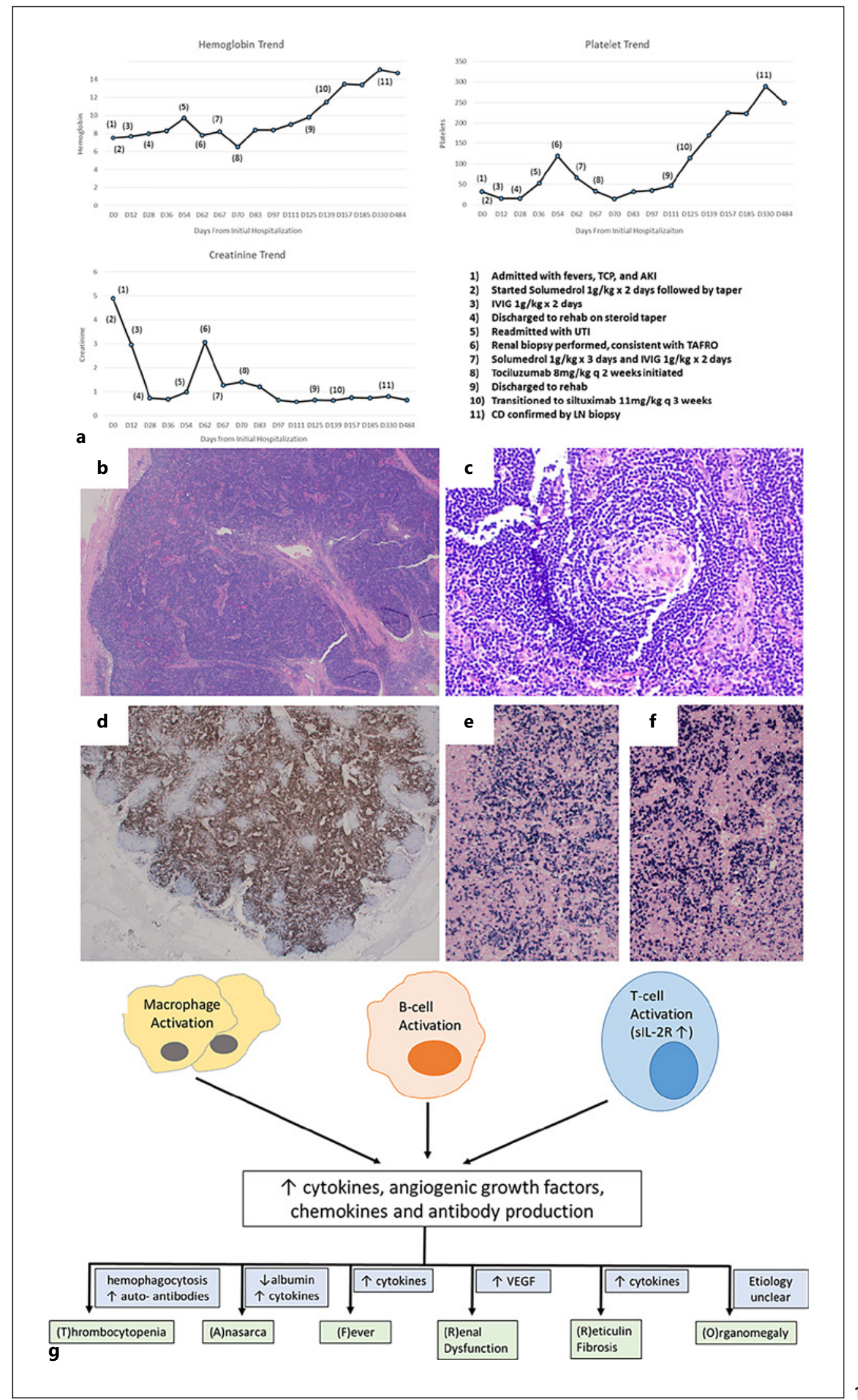


In cases such as ours, there may be inability to obtain tissue to promptly diagnose iMCD, which can cause delays in life-saving treatments. Our patient met all diagnostic criteria for TAFRO (3 major criteria of anasarca, thrombocytopenia, and fevers as well as 2 minor criteria of organomegaly and progressive renal insufficiency). However, she did not meet criteria for iMCD-TAFRO solely due to lack of LN tissue consistent with CD. In this setting, clinicians should recognize additional features that support underlying iMCDTAFRO, namely, elevated alkaline phosphatase, normal immunoglobulin levels, increased CRP/ESR, and hypoalbuminemia. Our patient exhibited these features, consistent with CD, as well as signs and symptoms of hypercytokinemia, and was empirically treated with IL-6 therapy with improvement in clinical course.

Our experience supports the use of empiric treatment with anti-IL 6 therapy in patients with TAFRO in the absence of a definitive diagnosis of IMCD; iMCD should be presumed, and anti-IL 6 therapy such as siltuximab should be considered in such cases, given its ease of administration and safety. Furthermore, such measures may help prevent rapid decompensation, allow time for patient stabilization to obtain definitive biopsy, potentially spare chemotherapy, and ultimately secure improvements in morbidity and mortality.

\section{Statement of Ethics}

Written informed consent was obtained from the patient for publication of this case report and any accompanying images. Information revealing the subject's identity was avoided. For this single case report, an ethical review board was not utilized per institutional guidelines.

\section{Conflict of Interest Statement}

C.W.: speakers bureau - AstraZeneca; R.K.: speakers bureau - AstraZeneca, Beigene, Kite/Gilead; consultant-Kite/Gilead, BMS/Juno, Karyopharm, Janssen, Morhphosys; research funding - Kite/Gilead, BMS/Juno, Takeda; D.C.F.: research funding - Janssen, EUSA Pharma; serves on the Board of Directors for the Castleman Disease Collaborative Network.

\section{Funding Sources}

No funding was provided for this case report.

\section{Author Contributions}

All authors participated in manuscript preparation and approved the final draft. L.B.S. provided histopathology pictures.

\section{Data Availability Statement}

All data on which the conclusions of the paper rely are available upon request to the corresponding author.

\section{Karger's}




\section{References}

1 Fajgenbaum DC. Novel insights and therapeutic approaches in idiopathic multicentric Castleman disease. Blood. 2018 Nov 30;132(1):2323-30.

2 van Rhee F, Voorhees P, Dispenzieri A, Fosså A, Srkalovic G, Ide M, et al. International, evidence-based consensus treatment guidelines for idiopathic multicentric Castleman disease. Blood. 2018 Nov 15;132(20): 2115-24.

3 Fajgenbaum DC, van Rhee F, Nabel CS. HHV-8-negative, idiopathic multicentric Castleman disease: novel insights into biology, pathogenesis, and therapy. Blood. 2014 May 8;123(19):2924-33.

4 Igawa T, Sato Y. TAFRO syndrome. Hematol Oncol Clin North Am. 2018 Feb;32(1):107-18.

5 Wang HW, Pittaluga S, Jaffe ES. Multicentric Castleman disease: where are we now? Semin Diagn Pathol. 2016 Sep;33(5):294-306.

6 Takai K, Nikkuni K, Momoi A, Nagai K, Igarashi N, Saeki T. Thrombocytopenia with reticulin fibrosis accompanied by fever, anasarca and hepatosplenomegaly: a clinical report of five cases. J Clin Exp Hematop. 2013; 53(1):63-8.

7 Sakashita K, Murata K, Takamori M. TAFRO syndrome: current perspectives. J Blood Med. 2018;9:15-23.

8 Iwaki N, Fajgenbaum DC, Nabel CS, Gion Y, Kondo E, Kawano M, et al. Clinicopathologic analysis of TAFRO syndrome demonstrates a distinct subtype of HHV-8-negative multicentric Castleman disease. Am J Hematol. 2016 Feb;91(2):220-6.

9 Fujimoto S, Sakai T, Kawabata H, Kurose N, Yamada S, Takai K, et al. Is TAFRO syndrome a subtype of idiopathic multicentric Castleman disease? Am J Hematol. 2019 Sep;94(9):975-83.

10 Yoshizaki K, Murayama S, Ito H, Koga T. The role of interleukin-6 in Castleman disease. Hematol Oncol Clin North Am. 2018 Feb;32(1):23-36.

11 Kurose N, Mizutani KI, Kumagai M, Shioya A, Guo X, Nakada S, et al. An extranodal histopathological analysis of idiopathic multicentric Castleman disease with and without TAFRO syndrome. Pathol Res Pract. 2019 Mar; 215(3):410-3.

12 van Rhee F, Wong RS, Munshi N, Rossi JF, Ke XY, Fosså A, et al. Siltuximab for multicentric Castleman's disease: a randomised, double-blind, placebo-controlled trial. Lancet Oncol. 2014 Aug;15(9):966-74. 